\title{
Expected number of Extensive Air Showers observable by EUSO-SPB
}

\author{
Francesco Fenu*, Mario Bertaina, Alberto Bortone, Andrea Veneziani, Simone \\ Cambursano \\ Università degli studi di Torino, INFN Torino, Italy \\ E-mail: francesco.fenu@gmail.com
}

\section{Austin Cummings}

Colorado School of Mines, US

\section{Naoto Sakaki}

ICRR-University of Tokyo, Japan

\section{for the JEM-EUSO collaboration}

\begin{abstract}
EUSO-SPB (Extreme Universe Space Observatory - Super Pressure Balloon) is the first pathfinder mission of the JEM-EUSO program aiming at the detection of the fluorescence light emitted by Extensive Air Shower (EAS) produced by cosmic ray particles in the atmosphere. EUSOSPB has flown in Spring 2017 from the Wanaka base in New Zealand on board a NASA Super Pressure Balloon planning to reach up to a 100 days of flight, giving, therefore, the opportunity to observe for the first time a bunch of cosmic ray events with the fluorescence technique from the edge of space. By means of the ESAF (EUSO Simulation and Analysis Framework) package, which is one of the official simulation tools to study the performance of the different pathfinder missions of the JEM-EUSO program, prior to flight, extensive simulations have been carried out to determine the expected number of detectable events as a function of several parameters related to the detector performance itself (i.e. trigger logic, optics and electronics efficiency), to the different environmental conditions (i.e. night-glow level, presence of clouds at different heights with variable optical depth) as well as to the launch season and duration of the flight. The main results of these simulation studies are presented.
\end{abstract}

35th International Cosmic Ray Conference - ICRC2017

10-20 July, 2017

Bexco, Busan, Korea

${ }^{*}$ Speaker. 


\section{Introduction}

The JEM-EUSO project [1] aims at the detection of Ultra High Energy Cosmic Rays from space. The instrument is designed to detect the fluorescence light emitted by cosmic ray showers in the atmosphere. In this framework several pathfinders have been developed or are in preparation. Between them we can cite EUSO-TA [2], EUSO-Balloon [3], Mini-EUSO [4] and EUSO-SPB [5]. The EUSO-SPB detector flew in April 2017 from the Wanaka base in New Zealand. Its main target was the detection of cosmic rays from the stratosphere.

EUSO-SPB further developed the concept of EUSO-Balloon, a JEM-EUSO prototype which flew in August 2014 from Timmins in Canada. This stratospheric flight lasted for one night and was testing for the first time the JEM-EUSO technology in near-space conditions. The EUSO-Balloon was not equipped with a self-trigger algorithm and was tested with a fixed acquisition rate and laser pulses from a helicopter. The measurement of the background was also a mission objective while no cosmic ray detection was expected [6].

EUSO-SPB was on the other hand designed to achieve 100 days of flight and to detect for the first time cosmic rays from the stratosphere through fluorescence. The detector was equipped with a fully autonomous trigger scheme and with solar panels to recharge batteries during daytime [7]. The payload had also antennas to transfer data on ground through satellite. The acquired data were temporarily stored on a hard disk before being sent to the control center. The daytime and moon time was used to download data.

We present here the methodology to derive through simulation the expected number of detectable events by EUSO-SPB. In general several factors are key players in the determination of the final estimation, among them: the detector performance; the trajectory of the balloon which is linked to the weather conditions; the duration of flight; the UV night-glow, which is seasonal and geographically variable. More in detail the detector performance includes: the overall efficiency of the instrument (i.e, optics throughput, photomultiplier response); the Point Spread Function (PSF) of the optics which is linked to the $S / N$ ratio; the trigger scheme which has the duty to make a massive screening of pixel responses to recognize signal excesses localized or following a track, as expected from an EAS, which lasts up to $20 \mu \mathrm{s}$. High trigger rates are also source of larger dead time for data acquisition, and in case of limited telemetry, like in SPB flights ( $\sim 100 \mathrm{kbit} / \mathrm{s})$, might impact on the data transfer. On the other hand balloon related aspects are: floating altitude, trajectory and duration of flight. The floating altitude is related to the Field of View (FoV), therefore, higher altitudes are preferable from this point of view but they correspond also to an increase of the energy threshold in detecting EAS as they would look dimmer. The duration of the night time is slightly shorter at higher altitudes. The trajectory of the balloon is another relevant parameter. The possibility to detect EAS requires either clear atmosphere or low clouds, such that shower maximum is visible and a track can be properly reconstructed. Middle clouds represent an obstacle for the observation of quasi-vertical EAS, while inclined ones are still detectable. High clouds block the observation of all types of EAS. Clouds are also linked to another important parameter which is the UV night-glow intensity. The reflected light from night-glow, air-glow or the zodiacal one, which can vary according to the season and geographical location impact on the energy threshold of the instrument, and, therefore, the possible number of events that can be detected. As a reference value the presence of clouds generates typically an increase by a factor of $\sim 2$ of the reflected UV 
light. The trajectory drives also the fraction of time in which the balloon floats on urbanized areas which prevents the observation of EAS. This fraction is, anyway, much less relevant in the southern hemisphere, but in principle not determined a priori, like in case of a satellite observation, as the balloon trajectory is subject to wind speed and direction.

In other words, a correct estimation of the expected number of events should take into account all these aspects, and some of them are known only after flight, when the detector performance in flight has been evaluated. For this reason we present here an estimation which is based on assumptions at the time in which the detector was still in the integration phase, and, therefore, borrowed from the EUSO-Balloon flight, and with the estimation of the cloud impact based on the previous SPB flights. In this sense, the results should be considered only as a guidance of the potential of the EUSO-SPB flight. Currently, ESAF code is being upgraded to take into account the real flight conditions to provide in future a more realistic estimation of the expected number of events. Anyhow, the methodology here described represents the basis of the analysis that should be conducted also in case of future EUSO balloon campaigns and for the main mission. A more detailed description of the results here presented can be found in [8] [9].

\section{Flight trajectory and estimation of the cloud fraction}

Three SPB flights have been conducted so far by NASA Balloon Facility from Wanaka New Zealand, one per year starting in 2015. Fig. 1 shows the trajectories and flight duration for all three flights. It is clear that the path of 2015 was significantly different from 2016.

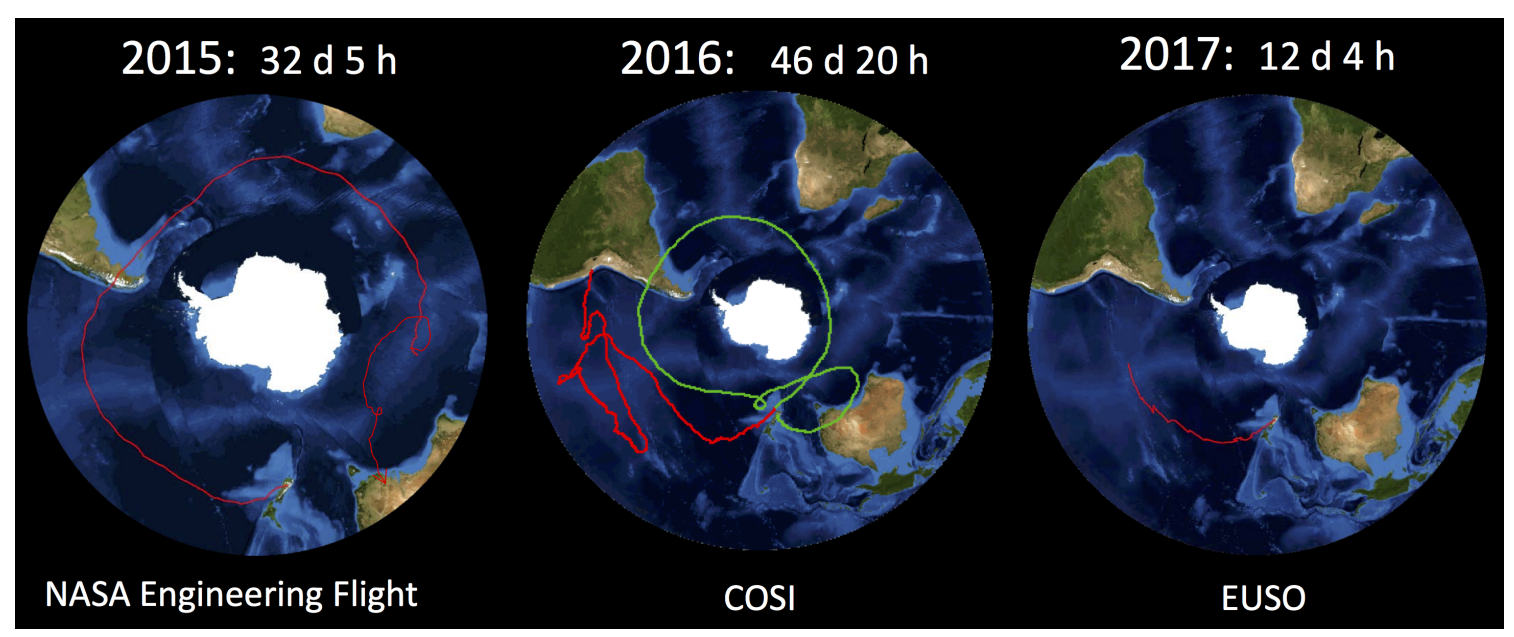

Figure 1: The SPB trajectories of the three flights.

In order to quantify the effect of cloud contamination, a study of the climatological distribution of clouds, as a function of cloud-top altitude, optical depth, and geographical location has been performed using the meteorological database ISCCP. The International Satellite Cloud Climatology Project (ISCCP) [10] was established in 1982 to collect and analyze satellite radiance measurements needed to infer the global distribution of clouds, their properties and their diurnal, seasonal and inter-annual variations. The ISCCP has developed cloud detection schemes using visible and IR window radiance (IR during nighttime and daytime, and visible during daytime). The 
data from 1983 to 2008 have been used in this analysis. Data are given on a 2.5 -degree grid in latitude and longitude. The fraction of clouds was computed by using the data in the catalogue on a band of 7.5 degrees around the trajectory. Table 1 shows the fraction of clear atmosphere, low, middle and high clouds for the two cases.

Table 1: Fraction of clouds in \% of different altitudes and of clear atmosphere along the trajectories of year 2015 and 2016. The reported uncertainty indicates the variability of the cloud fraction at different longitudes.

\begin{tabular}{|c|c|c|c|c|}
\hline flight & Clear Sky & Low Clouds $(\mathrm{h}<2 \mathrm{~km})$ & Middle Cl. $(2<\mathrm{h}<6 \mathrm{~km})$ & High Cl. $(\mathrm{h}>6 \mathrm{~km})$ \\
\hline 2015 & $23 \pm 7$ & $37 \pm 5$ & $25 \pm 4$ & $15 \pm 2$ \\
2016 & $40 \pm 2$ & $25 \pm 1$ & $22 \pm 1$ & $13 \pm 2$ \\
\hline
\end{tabular}

The average optical depth of the clouds was reported to be around $4.5 \pm 1.0$ for both cases. It is clear that the fractions of clear and low cloud sky remarkably depend on the flight trajectory, even though both situations are good for cosmic ray observation and they represent at least $60 \%$ of the fraction of time.

\section{The expected number of events}

The simulation software used in this study is the so called EUSO Simulation and Analysis Framework (ESAF) which is one of the JEM-EUSO simulation softwares [11] [12]. ESAF takes care of the simulation of all the physical processes involved in the detection of the cosmic ray. More in detail ESAF takes care of the development of the shower, of the generation of the fluorescence and Cerenkov photons and finally of the simulation of the detector itself.

EUSO-SPB is a refractive telescope based on a set of lenses of $1 \mathrm{~m}^{2}$ each. Light is focused on a Focal Surface (FS) made of 36 Multi-Anode Photo-Multipier Tubes (MAPMTs) of 64 pixels each (pixel size is $3 \mathrm{~mm}$ ). In the present study we assumed the design configuration of 3 lenses with an optical PSF of $\sim 1$ pixel and an overall throughput of 5\%, while in reality the instrument flew with only 2 lenses, a higher overall efficiency $(9 \pm 1) \%$ even though with a larger PSF $(\sim 3$ pixels).

A $\sim 20 \%$ non-uniform FS in detection efficiency was considered. Data are acquired in temporal frames of $2.5 \mu \mathrm{s}$, named Gate Time Units (GTUs). The trigger scheme adopted the single GTU First Level Trigger (see [13] for details) which means that the trigger required a signal excess on the background fluctuations lasting only 1 GTU in a box of $3 \times 3$ pixels. EUSO-SPB in flight used not only this configuration, but tested also other two conditions, in which the excess should last 2 or 5 GTUs to issue a trigger.

The floating altitude was assumed to be constant and two cases were considered to provide an uncertainty on this factor: a high altitude of $38 \mathrm{~km}$, as for the Timmins flight, and a lower one of 30 $\mathrm{km}$. Typical SPB floating altitude is $33 \mathrm{~km}$. In reality EUSO-SPB, after three days of floating at nominal altitude was subject to day and night variations with minima also around $\sim 20 \mathrm{~km}$ altitude.

The UV intensity in clear sky conditions was assumed to be similar to EUSO-Balloon flight which is 0.63 counts pixel ${ }^{-1} \mathrm{GTU}^{-1}$. The UV intensity above clouds was assumed to be roughly double of what measured in clear sky, again as a result of the EUSO-Balloon campaign. We 
simulated four different cloud conditions namely: clear sky, low clouds at $2 \mathrm{~km}$ altitude, middle clouds at $5 \mathrm{~km}$, high clouds at $10 \mathrm{~km}$ as representatives of the different conditions. We simulated only thick clouds with $\mathrm{OD}=5$ following the results on ISCCP analysis.

Regarding the total dark time, in the simulations we assumed three different cases. In the first calculation, the dark period on the moon phase of March - April 2017 was used which corresponds to 118 hours. In the other two cases the dark time measured during the previous flight campaigns was considered: 138 and 211 hours, respectively.

The number of triggered events is calculated as product between the calculated exposure and the flux published by the Pierre Auger Collaboration [14]. In Eq. 3.1 we see the equation for the exposure $\mathscr{E}$ calculation.

$$
\mathscr{E}(E)=\langle\varepsilon\rangle(E) \int_{A_{\text {simu }}} d A \int_{\Omega} d \Omega \int_{t_{\text {acq }}} d t
$$

In this equation $A_{\text {simu }}$ is the injection area and $\Omega$ is the solid angle covered. The exposure is calculated integrating over all the possible times $t_{a c q}$ where the detector is acquiring data. The average efficiency $\langle\varepsilon\rangle(E)$ factor is calculated with a simulation which takes into account all the possible impact points, energy and directions of the showers (see Eq. 3.2). In Eq. 3.2 (x,y) are the coordinates of the impact point of the shower and $(\theta, \phi)$ represents the arrival direction of the particles. Given the small size of the field of view ( $\pm 4 \mathrm{~km}$ side) compared to the showers a very large $A_{\text {simu }}$ is needed ( $>50 \mathrm{~km}$ radius). The calculation of the efficiency over all the possible impact positions and directions is however computationally very heavy. We therefore exclude all the directions where the efficiency is guaranteed to be 0 . We defined a volume $\mathscr{V}$ ( $8 \mathrm{~km}$ radius) around the FoV where showers must pass before being fully simulated by ESAF. Showers not passing through this volume are guaranteed to never cross the FoV (which is much smaller) and therefore are not simulated. In this way we speed up the simulation by a factor 10 at least. The average efficiency is therefore

$$
\langle\varepsilon\rangle(E)=\frac{\int_{A_{\text {simu }}} d A \int_{\Omega} d \Omega \frac{N_{\text {trigg }}(E, x, y, \theta, \phi)}{N_{\text {simu }}(E, x, y, \theta, \phi)}}{\int_{A_{\text {simu }}} d A \int_{\Omega} d \Omega} k
$$

where $k$ represents the average correction factor for the efficiency taking into account the fraction of events passing through the volume $\mathscr{V}$ with respect to the events on the entire solid angle $N_{\text {test }}$ (see eq. 3.3).

$$
k=\frac{\int_{A_{\text {simu }}} d A \int_{\Omega} d \Omega \frac{N_{\text {simu }}}{N_{\text {test }}}}{\int_{A_{\text {simu }}} d A \int_{\Omega} d \Omega}
$$

The number of events is then calculated as

$$
N(E)=\mathscr{E}(E) \psi(E)
$$

where $\psi(E)$ is a fit on the spectrum published by Pierre Auger [14]. We see in Fig. 2 the triggered spectrum as expected by EUSO-SPB in clear sky and for a mission duration $t_{a c q}$ of 118 hours. The total number of events has been calculated integrating the number of events on the entire energy range. The spectrum is peaked at energies around $3 \times 10^{18} \mathrm{eV}$. It is important to underline that, despite the differences previously mentioned on the detector performance between assumptions 


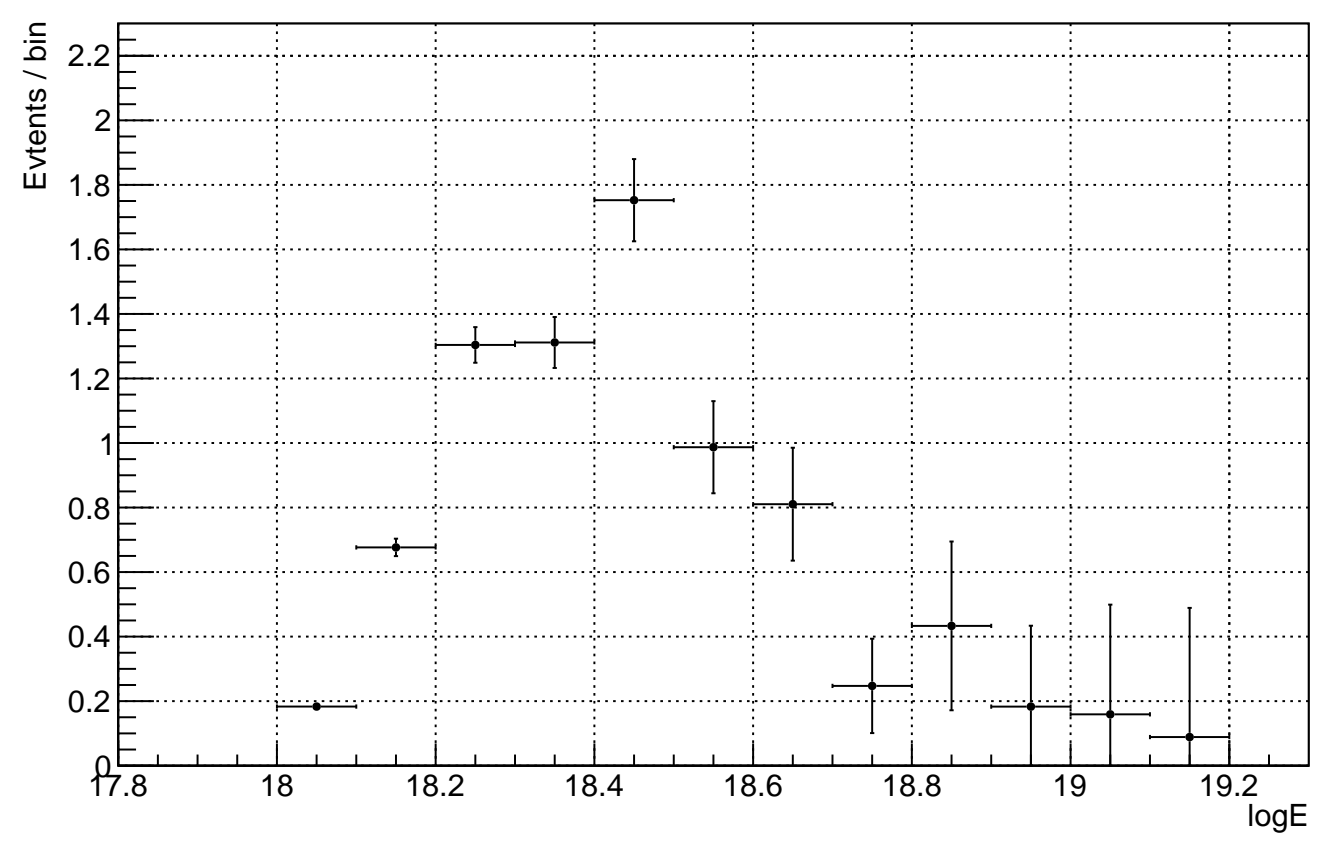

Figure 2: The expected number of triggered EAS floating at an altitude of $30 \mathrm{~km}$ assuming clear sky condition and a flight duration corresponding to 118 hours of dark time.

and real measurements, this is the energy at which EUSO-SPB resulted to be 50\% efficient in EAS triggering according to the field tests performed at EUSO-TA site in October 2016 [15].

All the cloud conditions have been simulated and for each of them the total number of triggered events has been calculated. Tab. 2 shows the expected integrated events in clear sky, low, middle and high clouds for a 118 hours dark time. Cloud condition affects in a significant way the number of detected cosmic rays.

Table 2: Number of expected particles detected in 118 hours of dark time. Four different representative sky conditions are simulated. Two balloon heights are considered.

\begin{tabular}{|c|c|c|c|c|}
\hline height & Clear Sky & Low Clouds $(2 \mathrm{~km})$ & Middle Clouds $(5 \mathrm{~km})$ & High Clouds \\
\hline $30 \mathrm{~km}$ & $8.0 \pm 0.6$ & $6.4 \pm 0.6$ & $4.9 \pm 0.5$ & 0 \\
$38 \mathrm{~km}$ & $3.8 \pm 0.5$ & $4.2 \pm 0.5$ & $3.2 \pm 0.4$ & 0 \\
\hline
\end{tabular}

A weighted sum of the results of tables 1,2 has been therefore performed in order to give an estimate of the expected rate in mixed cloud condition. The flight duration and trajectories of SPB flight 2015 and 2016 has been used. Results are shown in tab. 3.

Under these conditions we can conclude that EUSO-SPB had the potential to detect several EAS events on a nominal campaign of 100 days. Unfortunately the EUSO-SPB flight lasted only 12 days and the total number of collected hours of data are $\sim 30$. This includes the fact that the EUSO-SPB started its flight already during the moonless period and that in the first two nights it 
Table 3: number of expected particles assuming the 2015 and 2016 flights for two different balloon height.

\begin{tabular}{|c|c|c|c|}
\hline Year & Hours & $38 \mathrm{~km}$ height & $30 \mathrm{~km}$ height \\
\hline 2015 & 138 & $3.7 \pm 0.8$ & $6.3 \pm 0.9$ \\
2016 & 211 & $5.9 \pm 1.3$ & $10.6 \pm 2.3$ \\
\hline
\end{tabular}

was in commissioning phase in which the different instruments on board were under test.

A simple rescaling of the results of tab. 3 for a 30 hours flight would lead to a number of events ranging between $0.8-1.5$, which is at the limit of detection.

\section{Conclusions}

In the present study we described a methodology to determine the expected rate of cosmic rays on a wide range of possible conditions for the EUSO-SPB flight. The estimations presented in this work are based on the expected performance of the detector while it was still in its integration phase. The duration and cloud coverage were taken from the previous flights and from meteorological databases. Such estimations indicate that EUSO-SPB had the potential to detect several events on a nominal campaign of 100 days. Unfortunately, the very short duration of the flight (12 days) limits considerably the number of events that could be detected.

To give a more precise evaluation of the expected number of events the real detector performance as well as EUSO-SPB trajectory, presence of clouds and UV intensity have to be introduced in the simulations. In the collaboration efforts are therefore ongoing to establish the detector performance in flight $[7,16]$ and to study the cloud coverage both as measured by the on-board infrared camera [17] and by public databases. During the night the payload was descending down to $20 \mathrm{~km}$. Preliminary studies seem to indicate a slightly higher number of events at lower altitude. A devoted effort to better model the actual flight parameters in ESAF code is ongoing and will be presented soon.

\section{Acknowledgments}

This work was partially supported by Basic Science Interdisciplinary Research Projects of RIKEN and JSPS KAKENHI Grant (22340063, 23340081, and 24244042), by the Italian Ministry of Foreign Affairs and International Cooperation, by the Italian Space Agency through the ASI INFN agreement n. 2017-8-H.0, by NASA award 11-APRA-0058 in the USA, by the Deutsches Zentrum für Luft- und Raumfahrt, by the Helmholtz Alliance for Astroparticle Physics funded by the Initiative and Networking Fund of the Helmholtz Association (Germany), and by Slovak Academy of Sciences MVTS JEM-EUSO as well as VEGA grant agency project 2/0132/17. We acknowledge support from French space agency CNES. Russia is supported by the Russian Foundation for Basic Research Grant No 16-29-13065-ofi-m. The Spanish Consortium involved in the JEM-EUSO Space Mission is funded by MICINN \& MINECO under the Space Program projects: AYA2009-06037-E/AYA, AYA-ESP2010-19082, AYA-ESP2011-29489-C03, AYA-ESP2012-39115C03, AYA-ESP2013-47816-C4, MINECO/FEDER-UNAH13-4E-2741, CSD2009-00064 (Consolider 
MULTIDARK) and by Comunidad de Madrid (CAM) under projects S2009/ESP-1496 \& S2013/ICE2822.

\section{References}

[1] A. Santangelo et al., The JEM-EUSO mission: an introduction, Exp. Astron. (2015), 40:3-17, DOI $10.1007 / \mathrm{s} 10686-015-9482-\mathrm{x}$

[2] L. W. Piotrowski et al., (JEM-EUSO Coll.), The EUSO-TA detector: status and performance, Proc. of the ICRC2017 (2017)

[3] P. Von Ballmoos et al., The EUSO-Balloon pathfinder, Exp. Astron. (2015), 40:281-299, DOI $10.1007 / \mathrm{s} 10686-015-9467-9$

[4] M. Ricci et al., (JEM-EUSO Coll.), Mini-EUSO: a precursor mission to observe and study Atmosphere and Earth UV emission from the International Space Station, Proc of the ICRC2017 (2017)

[5] L. Wiencke, A. Olinto et al., (JEM-EUSO Coll.), EUSO-SPB Mission and Science, Proc. of the ICRC2017 (2017)

[6] M. Bertaina, P. Von Ballmoos et al., (JEM-EUSO Coll.), Results of the EUSO-Balloon flight, Proc. of the ICRC2017 (2017)

[7] S. Bacholle et al., (JEM-EUSO Coll.), The EUSO-SPB instrument, Proc. of the ICRC2017 (2017)

[8] A. Veneziani, Study on expected sky conditions during Super Pressure Balloon flights and observation of cosmic rays, Bachelor Thesis, Università degli Studi di Torino (2016).

[9] S. Cambursano, A simulation study of the EUSO-SPB trigger and reconstruction performance, Master Thesis, Università degli Studi di Torino (2016).

[10] http://isccp.giss.nasa.gov/

[11] C. Berat et al., ESAF: full simulation of space-based extensive air showers detectors, Astrop. Phys. 33(4), 221-247 (2010)

[12] F. Fenu, A simulation study of the JEM-EUSO mission for the detection of ultra-high energy Cosmic Rays, Doctoral Thesis (2013), Eberhard Karls Universität Tübingen

[13] G. Abdellaoui et al. (JEM-EUSO Coll.), Nucl. Instr. \& Meth. A, Vol. 866 (2017) p. 150.

[14] A. Schulz et al., (Pierre Auger Coll.), The measurement of the energy spectrum of cosmic rays above 3 $\times 10^{17} \mathrm{eV}$ with the Pierre Auger Observatory, Proc. of the ICRC2013 (2013)

[15] J. Bayer et al. (JEM-EUSO Coll.) "The trigger logic of EUSO-SPB and its performance", This Conference Proceedings, Busan (2017).

[16] J. Eser et al. (JEM-EUSO Coll.) "Preflight calibration and testing of EUSO-SPB in the lab and the desert", This Conference Proceedings, Busan (2017).

[17] L. Allen et al. (JEM-EUSO Coll.) "UCIRC: Infrared Cloud Monitor for EUSO-SPB", This Conference Proceedings, Busan (2017). 